Methods: Data were obtained from 670 patients with SLE from Department of Rheumatology and immunology in our hospital between January 2012 and October 2016.All patients fulfilled the 1997 ACR SLE classification criteria. Data about their gender, age, ethnic groups and result of antibodies test were collected. Results: Totally 670 completed data were collected.there are $438(65.37 \%)$ of these patients $(68.22 \%)$ were Han population,136 $(20.29 \%)$ were Uighur, 43 $(6.42 \%)$ were Kazakh, $30(4.48 \%)$ were hui people and 23 were other minorities live in xinjiang. The titer of ANA was higher in minorities than Han population ( $Z=-$ 3.516, $\mathrm{P}<0.01)$. As compared with Han population, the positive rate of anti-Smith antibaodies and anti-SSA antibodies in SLE patients, significantly higher than han population ( $\chi^{2}=5.902, P<0.017 ; \chi^{2}=42.787, P<0.001$, respectively). However, there are no significant different of the positive rate ofanti-SSA antibodies, anti-Scl-70 antibodies, anti-Jo-1 antibodies,anti-ds-DNA antibodies between Han population and minorities.

Conclusions: Distributions of antibodies in SLE patients were different between Han population and minorities.the positive rate of anti-Smith antibaodies and anti-SSA antibodies in SLE patients were significantly higher than han population. Disclosure of Interest: None declared

DOI: 10.1136/annrheumdis-2017-eular.6291

\section{AB1009 MRI CONTRIBUTES TO ACCURATE DIAGNOSIS OF NON-RADIOGRAPHIC AXIAL SPONDYLOARTHRITIS IN PATIENTS WITH SERUM NEGATIVE HLA-B27}

C.-C. Lu ${ }^{1}, 2$, G.-S. Huang ${ }^{3}$, H.-C. Chen ${ }^{1}$, T.S.-H. Lee ${ }^{4}$, F.-C. Liu ${ }^{1}$, S.-Y. Kao ${ }^{1}$, S.-J. Chu ${ }^{1}$, T.-Y. Ho ${ }^{1}$, C.-H. Chen ${ }^{5}$, S.-Y. Lyu ${ }^{6} .{ }^{1}$ Division of Rheumatology, Department of Internal Medicine, Tri-Service General Hopsital, National Defense Medical Center, Taipei City, Taiwan, Province of China; ${ }^{2}$ Department of Pathology, University of Washington, Seattle, United States; ${ }^{3}$ Department of Radiology, Tri-Service General Hopsital, National Defense Medical Center; ${ }^{4}$ Department of Health Promotion and Health Education, National Taiwan Normal University, Taipei City; ${ }^{5}$ Division of Rheumatology, Department of Internal Medicine, Taipei Tzu Chi Hospital, New Taipei City; ${ }^{6}$ Division of Radiology, Taoyuan General Hospital, Ministry of Health and Welfare, Taoyuan City, Taiwan, Province of China

Background: Based on ASAS axial spondyloarthritis $(\mathrm{SpA})$ criteria, the presence of structural changes of sacroiliac (SI) joints such as sclerosis, bone erosion, joint space widening or ankyloses dose not meet the definition of active sacroiliitis on magnetic resonance imaging (MRI), if there is no bone marrow edema (BME). However only less than half Asian patients with SpA were characterized by BME. Neither serum inflammatory markers such as $\mathrm{c}$ reactive protein (CRP) nor erythrocyte sedimentation rate (ESR) is able to be useful as diagnostic markers in the early phase of SpA. HLA-B27 is associated with early diagnosis of SpA and axial inflammation of SI joints on MRI. Nonetheless, HLA-B27 is not associated with structural lesions of SI joints. All factors contribute to difficult defining early Asian SpA patients in the absence of serum HLA-B27 and active imaging inflammation.

Objectives: The aim of this study is to evaluate the prevalence of structure changes of SI joints on MRI in Taiwanese SpA patients in the absence of serum HLA-B27.

Methods: Thirty-three patients with inflammatory back pain and morning stiffness (disease duration more than 3 months) and high disease activity (BASDAl $\geq 4$ ) who had to be either serum HLA-B27 positive (10 patients) with $\geq 1$ SpA-feature or HLA-B27 negative with $\geq 2$ SpA-features (22 patients) were included in this prospective study. All patients did not meet the definition for a positive radiograph according to the modified New York criteria. MRI was performed with multiple sequence (Coronal and axial T1-weighted spin echo, coronal and axial short-tau inversion recovery). SI joints were evaluated for the prevalence of subchondral BME and structure changes (sclerosis, bone erosion, joint space widening and ankylosis). All patients were tested for X-rays of the pelvis and serum levels of ESR and CRP. Correlation analysis was performed among the different collected variables.

Results: Subchondral BME was only present in 8 of 23 patients with SpA in the absence of serum HLA-B27 (34.8\%), while 7 of $10(70 \%)$ HLA-B27 serum positive $\mathrm{SpA}$ patients had active BME on MRI. Structural changes of SI joints, including sclerosis, bone erosion and joint space widening were identified in $8(80 \%), 10$ $(100 \%)$ and $5(50 \%)$ SpA patients with positive serum HLA-B27, respectively. Nevertheless, these structural changes of $\mathrm{SI}$ joints on MRI were more common in HLA-B27 serum negative patients, as $15(65.2 \%), 20(87.0 \%)$ and $7(30.4 \%)$ of 23 serum negative patients, respectively.

Conclusions: MRI contributes to detect structural changes of SI joints for patients with nonradiographic axial SpA in the absence of serum HLA-B27.

References:

[1] Rudwaleit, M. \& Sieper, J. Nat. Rev. Rheumatol. 2012;8, 262-268.

[2] van Tubergen, A. \& Weber, U. Nat. Rev. Rheumatol. 2012;8, 253-261.

[3] van der Heijde D, et al. Ann Rheum Dis 2017;0:1-14.

[4] Turina MC, et al. RMD Open 2017;2:e000319.

[5] Baraliakos, X. \& Braun, J. Nat. Rev. Rheumatol. 2013;9, 498-502.

[6] Navallas M, et al. Radiographics. 2013;33:933-56.

[7] Lambert RGW, et al. Ann Rheum Dis 2016;75:1958-1963.

[8] Chung HY, et al. Ann Rheum Dis. 2011;70:1930-6.

Disclosure of Interest: None declared

DOI: 10.1136/annrheumdis-2017-eular.6069

\section{AB1010 ULTRASOUND MORPHOSTRUCTURAL PATTERN OF THE TIBIOFIBULAR JOINT. PRELIMINARY RESULTS}

C. Hernández-Díaz ${ }^{1}$, L. Ventura-Rios ${ }^{1}$, M. Maldonado ${ }^{2}$, G. Sanchez-Bringas ${ }^{3}$. ${ }^{1}$ Laboratorio de Ultrasonido Musculoesquelético y Articular, Instituto Nacional de Rehabilitación, Mexico City, Mexico; ${ }^{2}$ Reumatologa, Cuzco, Peru; ${ }^{3}$ Departamento de Embriologia, Facultad de Medicina, UNAM, Mexico City, Mexico

Background: The anatomy of the proximal tibiofibular (TF) joint is directly related to its ability to withstand stress applied in either a longitudinal or axial fashion. It is traditionally evaluated by CT scan, however, in certain conditions, it could be evaluated by ultrasound (US) conveniently; so far US has not been using to evaluate the TF joint. US is an innocuous, accessible and cheap image technique, that has demonstrated its utility for evaluating joints in several pathologic conditions, and might have an important role in the early diagnosis of inflammatory, degenerative or even tumoral lesions at the level of the TF joint; there are no studies that have evaluated the morpho- structural pattern of the TF joint.

Objectives: To describe the morphostructural pattern of the tibiofibular joint in healthy subjects.

Methods: Subjects older than 18 yrs old, with no history of past/present lesion of the knee, without any joint or neurovascular disease were included. A short questionnaire related to physical activity applied, and clinical evaluation to discard instability performed. US of both knees in extension done, using an Esaote ${ }^{\circledR}$ MyLab 70 ultrasound equipment with a $7.5-12 \mathrm{MHz}$ linear transducer. Descriptive statistics done

Results: Thirty-six patients (27 women, $75 \%$ ) included, mean age $41.2 \pm 8.9$ years, mean weight $71 \pm 12.46 \mathrm{~kg}$, mean height $1.61 \pm 0.09 \mathrm{~ms}$ and BMI $71.07 \pm 12.40 .69 \%$ of the subjects practice mild exercise activities. By US mean distance between tibia and fibula were $3.2 \pm 1.17 \mathrm{~cm}$; the mean thickness of the ligaments (superior and inferior) was $3.2 \pm 0.99 \mathrm{~cm}$ and $3.2 \pm 0.89$ respectively, and in the superior e inferior fibular ligaments were $3.2 \pm 0.99 \mathrm{~cm}$ and $3.2 \pm 0.89 \mathrm{~cm}$ respectively. Ligaments were hyperechoic in $61.1 \%$, a well-defined border was seen only in $48.6 \%$. Inside of joints a hypoechoic tissue was observed

Conclusions: These preliminary results suggest that US can be a useful tool for evaluating the tibiofibular joint.

Disclosure of Interest: None declared

DOI: 10.1136/annrheumdis-2017-eular.6840

\section{AB1011 RELIABILITY OF SONOGRAPHIC PERITENON EXTENSOR TENDON INFLAMMATION PATTERN IN PSORIATIC ARTHRITIS}

C. Macía $^{1}$, H.B. Hammer ${ }^{2}$, S. Falcao ${ }^{3}$, J. Medina ${ }^{4}$, M. Gutierrez ${ }^{5}$, E. De Miguel $^{6} .{ }^{1}$ Rheumatology, Hospital Universitario Severo Ochoa, Madrid, Spain; ${ }^{2}$ Rheumatology, Diakonhjemmet Hospital, Oslo, Norway; ${ }^{3}$ Rheumatology, Chronic Diseases Study Center (CEDOC), NOVA Medical School, UNL, Lisboa, Portugal; ${ }^{4}$ Rheumatology, Hospital Clínico Universitario, Valladolid, Spain; ${ }^{5}$ Rheumatology, Division of Musculoskeletal and Rheumatic Disorders. Instituto Nacional de Rehabilitación, Mexico City, Mexico; ${ }^{6}$ Rheumatology, Hospital Universitario la Paz, Madrid, Spain

Background: Preliminary results demonstrate that the peritenon inflammation of the extensor digitorum tendon (PTI) is a specific ultrasound (US) pattern of Psoriatic Arthritis (PsA) at metacarpophalangeal joint (MCPj) level. It was suggested as a pattern playing a key role in the differential diagnosis between Rheumatoid Arthritis and PsA $(1,2)$.

Objectives: In spite of PTI's clinical impact, there are no data regarding the reliability of US PTI evaluation. The present study addressed this topic by testing the reliability of US on evaluation of PTI.

Methods: 27 consecutive non selected PsA patients with clinical involvement of at least one 2nd to 5th MCPj were included. A rheumatologist trained in PTI assessments obtained the US images exploring the dorsal aspect of MCPj from 2nd to 5th of both hands using a MyLab 70 XVG machine, Esaote, Genova, Italy, with a greyscale (GS) $13 \mathrm{MHz}$ probe and a $7.1 \mathrm{MHz}$ power Doppler (PD) frequency, PRF $750 \mathrm{~Hz}$ and a 60 Gain. 3-5 seconds videos of each MCPj were obtained in transversal and longitudinal views for further reliability analysis. In the inter-reader reliability analysis, performed by five readers from five different hospitals and four countries, it was scored as present or absent 1) PTI (defined as an hypoechoic swelling of the soft tissue surrounding the extensor tendon at MCPj level with or without PD) and 2) intra-articular synovitis (IAS, OMERACT definition), both in PD and GS. The consensus of true US results for every joint and lesion was achieved when at least three readers had the same opinion. Cohen's Kappa test was used for statistical analysis.

Results: Clinical MCPj involvement was present in 60 (27.7\%) of the 216 joints whereas US detected IAS and/or PTI in $75(34.7 \%)$. US showed GS PTI in 41 $(19 \%)$ and PD in $38(17.6 \%)$ of the joints, while GS IAS was found in $63(29.2 \%)$ with PD activity in $41(19 \%)$ of the joints. The inter-reader reliability is shown in the Table. Intra-reader reliability results expressed as mean Kappa were 0.826 for PTI PD, 0.784 for PTI GS, 0.743 for IAS PD and 0.637 for IAS GS.

Conclusions: US examination of MCPj shows that PTI is near as frequent as IAS in PsA with reliability of its scoring at least as good as for joint synovitis. This opens the possibility of introducing US scoring of PTI, which may be of importance for the diagnosis and follow-up of PsA patients.

References:

[1] Gutierrez M et al. Ann Rheum Dis 2011; 70: 1111-4. 
Table 1. Inter-reader reliability

\begin{tabular}{lccccl}
\hline PTI PD & Reader 2 & Reader 3 & Reader 4 & Reader 5 & Mean Kappa 0.685 \\
\hline Reader 1 & 0.755 & 0.887 & 0.772 & 0.641 & \\
Reader 2 & & 0.705 & 0.666 & 0.534 & \\
Reader 3 & & & 0.722 & 0.644 & \\
Reader 4 & & & & 0.525 & \\
\hline PTI GS & Reader 2 & Reader 3 & Reader 4 & Reader 5 & Mean Kappa 0.590 \\
\hline Reader 1 & 0.682 & 0.778 & 0.739 & 0.535 & \\
Reader 2 & & 0.608 & 0.603 & 0.456 & \\
Reader 3 & & & 0.614 & 0.445 & \\
Reader 4 & & & & 0.440 & \\
\hline IAS PD & Reader 2 & Reader 3 & Reader 4 & Reader 5 & Mean Kappa 0.680 \\
\hline Reader 1 & 0.562 & 0.733 & 0.685 & 0.763 & \\
Reader 2 & & 0.597 & 0.590 & 0.714 & \\
Reader 3 & & & 0.640 & 0.806 & \\
Reader 4 & & & & 0.706 & \\
\hline IAS GS & Reader 2 & Reader 3 & Reader 4 & Reader 5 & Mean Kappa 0.567 \\
\hline Reader 1 & 0.593 & 0.593 & 0.511 & 0.727 & \\
Reader 2 & & 0.583 & 0.478 & 0.615 & \\
Reader 3 & & & 0.423 & 0.579 & \\
Reader 4 & & & & 0.564 & \\
\hline
\end{tabular}

[2] Zabotti A et al. Clinical and Experimental Rheumatology 2016; 34: 459-465. Disclosure of Interest: None declared

DOI: 10.1136/annrheumdis-2017-eular.1438

\section{AB1012 CLINICAL UTILITY OF ANTIHISTONE ANTIBODIES: A DESCRIPTIVE STUDY}

D. Boveda-Ruiz ${ }^{1}$, B. Vilas-Pio ${ }^{2}$, M. Freire-Dapena ${ }^{2}$, R. Lorenzo-Castro ${ }^{2}$, A. Soto-Peleteiro $^{2}$, S. Mirete-Bachiller ${ }^{3}$, A. Rivera-Gallego ${ }^{2}{ }^{1}$ Internal Medicine, Hospital Clínico Universitario de Valladolid (SACYL), Valladolid; ${ }^{2}$ Internal Medicine; ${ }^{3}$ Immunology, Complexo Hospitalario Universitario de Vigo, Vigo, Spain

Background: Antihistone antibodies (AHA) have been linked to Drug-Induced Lupus Erythematosus (DILE) for decades ${ }^{1}$. However, for some authors this relationship is not so clear and sugest that the presence of these autoantibodies is related to other autoimmune diseases more frequently $y^{2,3,4}$.

Objectives: The main objetive of this work was to study the association of AHA with different autoimmune entities (including DILE) and secondarily, look into which clinical manifestations and which autoantibodies are more frequently related to AHA.

Methods: We performed a descriptive study. A database was constituted using all patients with $\mathrm{AHA}+$ in any blood analysis between years 2000 and 2016 in the University Hospital Complex of Vigo. The variables of the study were: presence of autoimmune disease, clinical manifestations and related autoantibodies. Results:

\begin{tabular}{lcccc}
\hline Variable & Men & Women & All & $\%$ Total \\
\hline Age & 50 & 45 & 46 & \\
Gender & 15 & 58 & 73 & 100 \\
SLE & 8 & 27 & 35 & 48 \\
DILE & 0 & 0 & 0 & 0 \\
Scleroderma & 0 & 4 & 4 & 5 \\
Sjögren & 1 & 8 & 9 & 12 \\
Rheumatoid Arthritis & 0 & 3 & 3 & 4 \\
No diagnosis & 5 & 19 & 24 & 33 \\
Malar rash & 1 & 11 & 12 & 16 \\
Photosensitivity & 2 & 11 & 13 & 18 \\
Oral ulcers & 1 & 10 & 11 & 15 \\
Arthritis & 6 & 31 & 37 & 51 \\
Lupic nephropathy & 4 & 13 & 17 & 23 \\
Raynaud & 1 & 11 & 12 & 16 \\
Hematological abnormalities & 5 & 20 & 25 & 34 \\
AntiRo+ & 4 & 12 & 16 & 22 \\
AntiLa+ & 2 & 5 & 7 & 10 \\
AntiSm+ & 3 & 8 & 11 & 15 \\
AntiDNAds+ & 3 & 25 & 28 & 38 \\
\hline
\end{tabular}

None of the 73 patients AHA+ developed DILE while almost the $50 \%$ of them suffer any other autoimmune disease. We found a high percentage of $\mathrm{AHA}+$ patients with lupus erythematous complications such as arthritis and hematological abnormalities. AntiDNAds antibody was the more frequent coexpressed autoantibody.

Conclusions:

- AHA detection is not useful as DILE screening

- $A H A+$ sugest the presence of other autoimmune disease rather than DILE.

- AHA+ may be related to lupus erythematous systemic complications.

References:

[1] Fritzler MJ, Tan EM. Antibodies to histone in drug-induced and idiopathic lupus erythematosus. J Clin Invest. 1978;62:560-567.

[2] Peng SL, Craft J. Antinuclear antibodies. In: Ruddy S, Harris ED, Sledge CB, eds. Kelley's Textbook of Rheumatology. 6th ed. Philadelphia, Pa: WB Saunders; 2001:166.
[3] Kubo $\mathrm{M}$, Ihn H, Yasawa $\mathrm{N}$, et al. Prevalence and antigen specificity of antihistone antibodies in patients with polymyositis/dermatomyositis. J Invest Dermatol. 1999;112:711-715.

[4] Zirwas MJ, Kress DW, Deng JS. The Utility of Antihistone Antibody Screening in the Diagnosis of Drug-Induced Lupus Erythematosus. Arch Dermatol Apr 2004; VOL 140: 494-495.

Disclosure of Interest: None declared

DOI: 10.1136/annrheumdis-2017-eular.3005

\section{AB1013 DEVELOPMENT AND VALIDATION OF A FLOURESCENCE OPTICAL IMAGING RHEUMATOID ARTHRITIS SCORING SYSTEM FOR SYNOVITIS IN THE WRIST AND HAND}

D. Glinatsi ${ }^{1}$, M. Østergaard ${ }^{1,2}$, L. Terslev ${ }^{3}$, S. Ohrndorf ${ }^{4}$,

M. Ammitsbøll-Danielsen ${ }^{3} .{ }^{1}$ Copenhagen Center for Arthritis research, COPECARE, Center for Rheumatology and Spine Diseases, Rigshospitalet, Glostrup; ${ }^{2}$ Department of Clinical Medicine, Faculty of Health and Medical Sciences, University of Copenhagen, Copehangen; ${ }^{3}$ Copenhagen Center for Arthritis Research (COPECARE), Center for Rheumatology and Spine Diseases, Rigshospitalet, Glostrup, Denmark; ${ }^{4}$ Department of Rheumatology and Clinical Immunology, Charité - University Medicine, Berlin, Germany

Background: Fluorescence optical imaging (FOI) has been suggested as an imaging modality for assessment of inflammation (i.e. synovitis) in the hands, and has in several studies been compared to US and MRI, using different, but not validated scoring systems.

Objectives: To develop and validate a semi-quantitative FOI RA scoring system for synovitis in the wrist and hand.

Methods: 46 RA patients, eligible for induction or intensification of disease modifying anti-rheumatic drug and with $>1$ clinically swollen joint in the hand were included. FOI image-sets of both wrists and hands were obtained at baseline, and after 3 and 6 months' follow-up using a Xiralite system unit (nanoPET Pharma GmbH, Berlin, Germany). The patients received a bolus of i.v. indocyanine green (ICG) pulsion (1 $\mathrm{mg} / \mathrm{kg}$ body weight) 10 seconds after starting the examination, which obtained 1 image/second over 6 minutes. The image-sets were anonymized and randomized and were assessed for synovitis at the wrist, 1st-5th metacarpophalangeal, 1st interphalangeal and 2nd-4th proximal interphalangeal joint levels in both hands by two readers blinded to patient data but not chronology. 23 image-sets were re-anonymized and re-read for intrareader agreement analysis. The scoring system for synovitis was based on the assumption that inflamed tissue would demonstrate a more rapid enhancement than surrounding tissues. For each joint, the images were assessed sequentially from start of the injection of ICG-pulsion to peak enhancement. At the peak enhancement, the color index was adjusted in order to increase the discrepancy between colors. Synovitis was defined as a sharply marginated enhancement with clear delineation from surrounding tissues and correct anatomical location lasting $\geq 3$ seconds. The thickness of the pathology fulfilling these criteria was compared to the width of the joint in the transverse plane at the 3rd second of enhancement and the following semi quantitative scoring system $(0-3)$ was applied: grade 0 : no enhancement, grade $1:<1 / 3$, grade $2: \geq 1 / 3$ but ?2/3, grade $3:>2 / 3$ of joint thickness (range 0-66). Descriptive statistics and the Wilcoxon signed-rank test were used to assess change in score over time. Intra-/inter-reader for status and change scores were assessed using single measure intra-class correlation coefficients (ICC) and smallest detectable change (SDC, change scores only). Responsiveness was assessed using standardized response mean (SRM).

Results: Median (IQR) total synovitis score at baseline was $9.5(4.0 ; 16.5)$ and improved with $-5.0(-10.0 ;-1.0)$ and $-8.0(-13.5 ;-3.0)$ at 3 and 6 months' follow up, respectively $(p<0.01)$. Intra- and inter-reader ICCs were good to very good for total scores (Table 1). The SDCs were generally low and for the inter-reader SDCs, $56 \%$ and $60 \%$ of the patients had a change $>$ SDC between baseline and 3 and 6 months, respectively. The mean SRM for total change scores at 3 at 6 months' follow-up were moderate to good (0.7 and 0.8).

\begin{tabular}{|c|c|c|c|c|c|c|c|}
\hline & $\begin{array}{c}\text { ICC } \\
\text { Baseline }\end{array}$ & $\begin{array}{c}\text { ICC } \\
3 \text { months' } \\
\text { follow-up }\end{array}$ & $\begin{array}{c}\text { ICC } \\
6 \text { months' } \\
\text { follow-up }\end{array}$ & $\begin{array}{c}\text { ICC } \\
\text { Change, } \\
\text { baseline to } \\
3 \text { months }\end{array}$ & $\begin{array}{c}\text { ICC } \\
\text { Change, } \\
\text { baseline to } \\
6 \text { months }\end{array}$ & $\begin{array}{c}\text { SOC } \\
\text { Change, } \\
\text { baseline } \\
\text { to } \\
3 \text { months }\end{array}$ & $\begin{array}{c}\text { SOC } \\
\text { Change, } \\
\text { baseline } \\
\text { to } \\
6 \text { months }\end{array}$ \\
\hline & \multicolumn{7}{|c|}{ Intra-reader agreement, reader 1} \\
\hline Total scores & 0.90 & 0.86 & 0.83 & 0.87 & 0.92 & 6.2 & 4.9 \\
\hline Wrist & 0.82 & 0.92 & 0.62 & 0.88 & 0.91 & 1.4 & 1.4 \\
\hline MCP joints & 0.86 & 0.72 & 0.93 & 0.76 & 0.85 & 4.8 & 3.6 \\
\hline \multirow[t]{2}{*}{ PIP joints } & 0.95 & 0.96 & 0.86 & 0.90 & 0.90 & 2.9 & 3.3 \\
\hline & \multicolumn{7}{|c|}{ Intra-reader agreement, reader 2} \\
\hline Total scores & 0.95 & 0.97 & 0.90 & 0.90 & 0.90 & 6.2 & 6.5 \\
\hline Wrist & 0.92 & 0.88 & 0.81 & 0.78 & 0.80 & 1.6 & 1.6 \\
\hline MCP joints & 0.96 & 0.96 & 0.92 & 0.92 & 0.89 & 3.3 & 3.4 \\
\hline \multirow[t]{2}{*}{ PIP joints } & 0.94 & 0.95 & 0.80 & 0.88 & 0.90 & 3.8 & 3.9 \\
\hline & \multicolumn{7}{|c|}{ Inter-reader agreement } \\
\hline Total scores & 0.88 & 0.84 & 0.60 & 0.81 & 0.70 & 4.8 & 6.2 \\
\hline Wrist & 0.76 & 0.72 & 0.58 & 0.67 & 0.65 & 1.1 & 1.3 \\
\hline MCP joints & 0.86 & 0.80 & 0.76 & 0.81 & 0.66 & 3.1 & 3.7 \\
\hline PIP joints & 0.91 & 0.82 & 0.39 & 0.79 & 0.71 & 2.6 & 3.6 \\
\hline
\end{tabular}
very good: $I C C 20.80$. Abbreviations: SDC: smallest detectable change, MCP: metacarpophalangeal, PIP: proximal interphalangeal. 